\title{
Near-Field Spherical Scanning Antenna Measurements: Probe Deconvolution and Sensitivity
}

\author{
Ronald C. Wittmann and Michael H. Francis \\ National Institute of Standards and Technology, Boulder, CO 80305, USA \\ Correspondence should be addressed to Ronald C. Wittmann; wittmannrc@gmail.com \\ Received 5 August 2014; Revised 30 October 2014; Accepted 16 November 2014; Published 4 December 2014 \\ Academic Editor: Miguel Ferrando Bataller \\ Copyright ( 2014 R. C. Wittmann and M. H. Francis. This is an open access article distributed under the Creative Commons \\ Attribution License, which permits unrestricted use, distribution, and reproduction in any medium, provided the original work is \\ properly cited.
}

\begin{abstract}
We define and calculate sensitivity for several actual and simulated probes. Probe sensitivity can have a significant impact on the measurement uncertainty associated with probe deconvolution in near-field spherical-scanning, antenna measurements.
\end{abstract}

\section{Introduction}

In near-field planar scanning, we know that if the receiving pattern of the probe has a null in a given direction, then the far-field pattern of the test antenna cannot be determined reliably in that direction [1]. That is, the probe must be sensitive to plane-wave modes of interest. On the other hand, in near-field spherical scanning, there is no direct relationship between the probe and the test antenna patterns in a given direction. Nevertheless, we argue that the probe must be sensitive to all spherical-wave modes needed to represent the radiated fields.

This paper presents a small selection of results from a larger study of probe-sensitivity issues in external and internal spherical-scanning measurements. We calculate and discuss probe (modal) sensitivity as an aid in the choice of probes and measurement parameters (this is an expanded version of a paper presented at the 2012 Antenna Measurements Techniques Association Symposium [2]).

\section{External Scanning}

In external spherical scanning, we determine the radiated fields outside of the measurement sphere that are due to sources confined within the measurement sphere (see Figure 1). This is commonly called spherical scanning without the qualifier "external." The theory presented here is a synopsis with little detail beyond that needed for understanding this paper. A complete discussion may be found in [3]. by

The far-field radiation of an antenna can be characterized

$$
\mathbf{E}(\mathbf{r}) \underset{r \rightarrow \infty}{\sim} \mathbf{t}(\widehat{\mathbf{r}}) \frac{\exp (i k r)}{i k r} a_{0} .
$$

This formula embodies the linear relationship between the radiated electric field $\mathbf{E}(\mathbf{r})$ and the excitation $a_{0}$. The transmission function $\mathbf{t}(\widehat{\mathbf{r}})$ may be expanded as

$$
\mathbf{t}(\widehat{\mathbf{r}})=\sum_{n=1}^{N} \sum_{m=-n}^{n}\left[t_{n m}^{1} \mathbf{X}_{n m}^{1}(\widehat{\mathbf{r}})+t_{n m}^{2} \mathbf{X}_{n m}^{2}(\widehat{\mathbf{r}})\right]
$$

where $\mathbf{X}_{n m}^{1}$ and $\mathbf{X}_{n m}^{2}=\hat{\imath} \widehat{\mathbf{r}} \times \mathbf{X}_{n m}^{1}$ are vector spherical harmonics [4, Chapter 16], which depend only on the direction $\widehat{\mathbf{r}}$, and the $t_{n m}^{\ell}$ are (unknown) modal coefficients.

The antenna under test (AUT) is characterized by measurement with a probe that moves over a spherical surface of radius $r$, enclosing the AUT. To account for polarization, two measurements are made for each probe location $\mathbf{r}$. These measurements correspond to probe orientations differing by a rotation of $90^{\circ}$ about the probe axis. We follow common practice and use a "special symmetric" or " $\mu= \pm 1$ " probe. For such probes, the polarization measurements are 


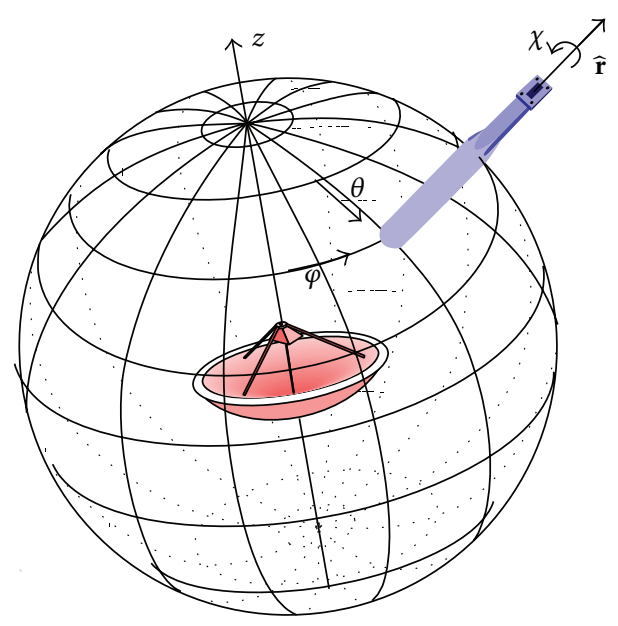

FIGURE 1: External spherical scanning.

the components of a transverse "measurement vector" $\mathbf{w}(\mathbf{r})$ that also may be expanded in spherical harmonics:

$$
\frac{\mathbf{w}(\mathbf{r})}{a_{0}}=\sum_{n=1}^{N} \sum_{m=-n}^{n}\left[T_{n m}^{1}(r) \mathbf{X}_{n m}^{1}(\widehat{\mathbf{r}})+T_{n m}^{2}(r) \mathbf{X}_{n m}^{2}(\widehat{\mathbf{r}})\right] .
$$

The relationship between the (known) $T_{n m}^{\ell}$ and the $t_{n m}^{\ell}$ is given by the probe-correction equations

$$
\left(\begin{array}{c}
T_{n m}^{1}(r) \\
T_{n m}^{2}(r)
\end{array}\right)=\mathbf{M}_{n}(r)\left(\begin{array}{c}
t_{n m}^{1} \\
t_{n m}^{2}
\end{array}\right)
$$

where

$$
\begin{aligned}
\mathbf{M}_{n}= & i \sqrt{\frac{4 \pi}{2 n+1}} \\
& \times\left(\begin{array}{cc}
\sigma_{n,-1}^{1}-\sigma_{n 1}^{1} & \sigma_{n,-1}^{2}-\sigma_{n 1}^{2} \\
-\sigma_{n,-1}^{1}-\sigma_{n 1}^{1} & -\sigma_{n,-1}^{2}-\sigma_{n 1}^{2}
\end{array}\right) .
\end{aligned}
$$

The translated probe coefficients $\sigma_{n m}^{\ell}$ are basically the responses of the probe, located at $r \widehat{\mathbf{z}}$, to each of the multipoles that compose the basis of vector spherical waves. Symbolically,

$$
\begin{aligned}
\sigma_{n m}^{1}(r) & =\left[\mathscr{P} \cdot \mathbf{m}_{n m}\right](r \widehat{\mathbf{Z}}), \\
\sigma_{n m}^{2}(r) & =\left[\mathscr{P} \cdot \mathbf{n}_{n m}\right](r \widehat{\mathbf{z}}),
\end{aligned}
$$

where the operator $\mathscr{P}$ denotes the probe and $\mathbf{m}_{n m}(\mathbf{r})$ and $\mathbf{n}_{n m}(\mathbf{r})$ represent magnetic and electric multipoles, respectively, that generate outgoing waves at infinity. For a special symmetric probe, $\sigma_{n \mu}^{\ell}=0$ unless $\mu= \pm 1$.

We consider linearly polarized probes with symmetries

$$
\sigma_{n,-1}^{1}=-\sigma_{n 1}^{1}, \quad \sigma_{n,-1}^{2}=\sigma_{n 1}^{2}
$$

that are actually satisfied by many practical probes. In this case $\mathbf{M}_{n}$ is diagonal and

$$
\begin{aligned}
& \left|T_{n m}^{1}(r)\right|=\alpha_{n}^{1}(r)\left|t_{n m}^{1}\right|, \\
& \left|T_{n m}^{2}(r)\right|=\alpha_{n}^{2}(r)\left|t_{n m}^{2}\right|,
\end{aligned}
$$

where the probe sensitivity is defined as

$$
\alpha_{n}^{\ell}=\sqrt{\frac{16 \pi}{2 n+1}}\left|\sigma_{n 1}^{\ell}\right| .
$$

In the general case, appropriate sensitivities may be defined as the singular values of $\mathbf{M}_{n}$ [5]. Of course, quantities corresponding to our definition of probe sensitivity are calculated, directly or indirectly, in any standard version of probe-corrected, near- to far-field transformation software.

When $\alpha_{n}^{\ell}$ is small, $t_{n m}^{\ell}$ is generally the ratio of two small numbers. As the signal falls towards the noise floor, the relative uncertainty in $T_{n m}^{\ell}$ and thus $t_{n m}^{\ell}$ can become extremely large. On the other hand, enhanced sensitivity to some modes is not necessarily beneficial. When the full dynamic range of the receiver is available, it is generally better to have uniform sensitivity over the modes of interest. The condition number

$$
c_{a}(r)=\frac{\max _{n, \ell}\left[\alpha_{n}^{\ell}(r)\right]}{\min _{n, \ell}\left[\alpha_{n}^{\ell}(r)\right]}
$$

is a good measure of merit; the optimum value is $c_{\alpha}=1$.

\section{Internal Scanning}

In internal spherical scanning, we determine the radiated fields within the measurement sphere that are due to sources located outside of the measurement sphere (see Figure 2). In the neighborhood of the coordinate origin (centered in the measurement sphere),

$$
\mathbf{E}(\mathbf{r})=\frac{a_{0}}{4 \pi} \int \mathbf{t}(\widehat{\mathbf{k}}) \exp (i \mathbf{k} \cdot \mathbf{r}) d \widehat{\mathbf{k}},
$$




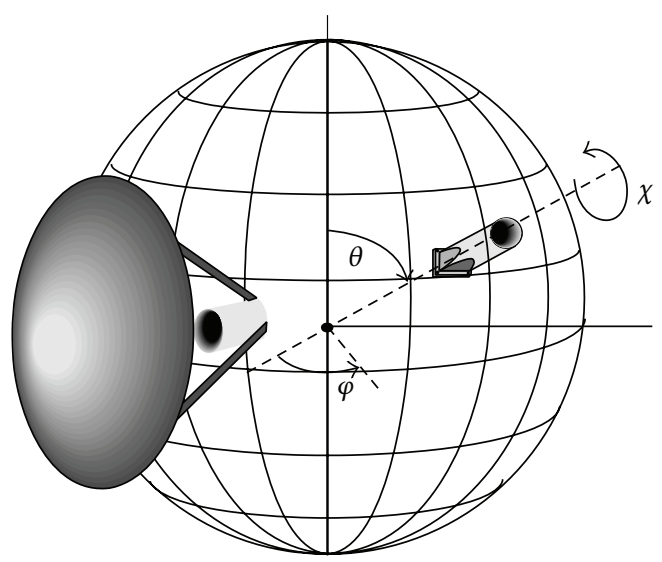

FIGURE 2: Internal spherical scanning.

where $\mathbf{t}(\widehat{\mathbf{k}})$ is the "transmitting function" of the sources. In principle, knowledge of $\mathbf{t}(\widehat{\mathbf{k}})$ allows compensation, say, of compact range measurements, for the effects of nonuniform illumination [6-8].

Equations (2)-(10) also apply to internal scanning, with the exception that (6) becomes

$$
\begin{aligned}
\sigma_{n m}^{1}(r) & =\left[\mathscr{P} \cdot \mathbf{m}_{n m}^{(1)}\right](r \widehat{\mathbf{Z}}), \\
\sigma_{n m}^{2}(r) & =\left[\mathscr{P} \cdot \mathbf{n}_{n m}^{(1)}\right](r \widehat{\mathbf{z}}),
\end{aligned}
$$

where $\mathbf{m}_{n m}^{(1)}(\mathbf{r})$ and $\mathbf{n}_{n m}^{(1)}(\mathbf{r})$ represent magnetic and electric multipoles, respectively, that remain finite at the origin. This "small" change has a profound effect on the nature of probe correction in the interior versus exterior case.

\section{Processing and Uncertainty}

Data typically are collected on a uniform grid in $\theta$ and $\varphi$ with sample increments chosen to satisfy the Nyquist sampling theorem

$$
\Delta \theta, \Delta \varphi \leq \frac{2 \pi}{2 N+1} .
$$

In the NIST algorithm, the angular measurements are transformed to a Fourier representation by use of the fast Fourier transform (FFT). Orthogonality integral relations are then employed to project the Fourier representation onto the basis of spherical harmonics, producing the representation (3). Finally, probe effects are removed through deconvolution to yield the far-field pattern (2).

The FFT is unitary so that the root mean square (RMS) error in the Fourier representation is the same as the RMS error in the measured data. The projection operation also will not increase the RMS error; in fact, there will be a reduction if some of the error signal lies in the null space of the projection. For the probe deconvolution, assuming that the uncertainty in sensitivity $\Delta \alpha_{n}$ is 0 , we have

$$
\frac{\|\Delta \mathbf{t}\|}{\|\mathbf{t}\|} \leq c_{\alpha} \frac{\|\Delta \mathbf{T}\|}{\|\mathbf{T}\|}
$$

(see (A.3)), where, for example,

$$
\|\mathbf{t}\|^{2}=\sum_{n=1}^{N} \sum_{m=-n}^{n}\left(\left|t_{n m}^{1}\right|^{2}+\left|t_{n m}^{2}\right|^{2}\right) .
$$

Thus, the use of a poor probe can lead to significantly expanded uncertainties.

We can also account for the fact that the sensitivities are not known precisely. With $\Delta T_{n}=0$

$$
\frac{\|\Delta \mathbf{t}\|}{\|\mathbf{t}\|} \leq \beta
$$

(See (A.5)) Here

$$
\beta \approx \max _{n, \ell}\left|\frac{\Delta \alpha_{n}^{\ell}}{\alpha_{n}^{\ell}}\right| .
$$

Equations (14) and (16) may be combined in quadrature to give an overall view of the effect of probe deconvolution on measurement uncertainty:

$$
\left(\frac{\|\Delta \mathbf{t}\|}{\|\mathbf{t}\|}\right)^{2} \lesssim\left(c_{\alpha} \frac{\|\Delta \mathbf{T}\|}{\|\mathbf{T}\|}\right)^{2}+\beta^{2} .
$$

Although illustrative and easy to derive, (18) gives a conservative estimate of uncertainty that can be refined.

NIST software calculates with double-precision accuracy to ensure that computational errors are negligible compared to measurement errors.

\section{Examples}

We consider a mode cut-off of $N=100$. This is sufficient for the representation of radiated fields when $k r_{0} \lesssim 100$. In the external case, $r_{0}$ is the radius of the minimum sphere of the test antenna. In the internal case, $r_{0}$ is the radius of the test zone. For practical reasons, the measurement radius $r_{\mathrm{m}}$ is usually chosen to be 3 or more wavelengths greater than $r_{0}$. The radius $r_{\mathrm{m}}$ with $k r_{\mathrm{m}}=105$ is just outside the sphere $r_{0}=100 / k$. The radius $r_{\mathrm{m}}$ with $k r_{\mathrm{m}}=200$ is greater than or equal to twice $r_{0}$. 


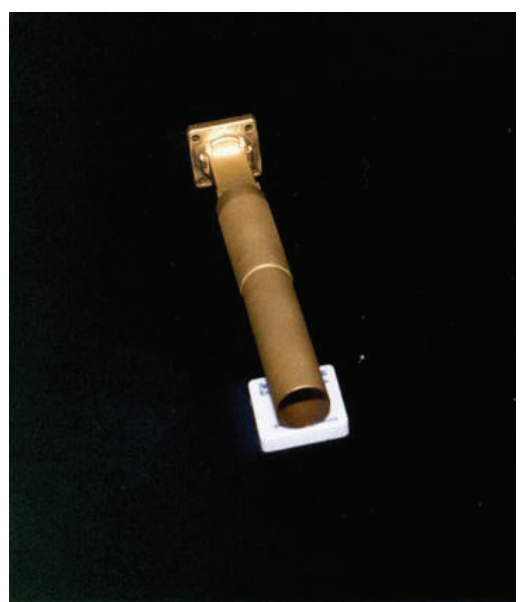

(a)

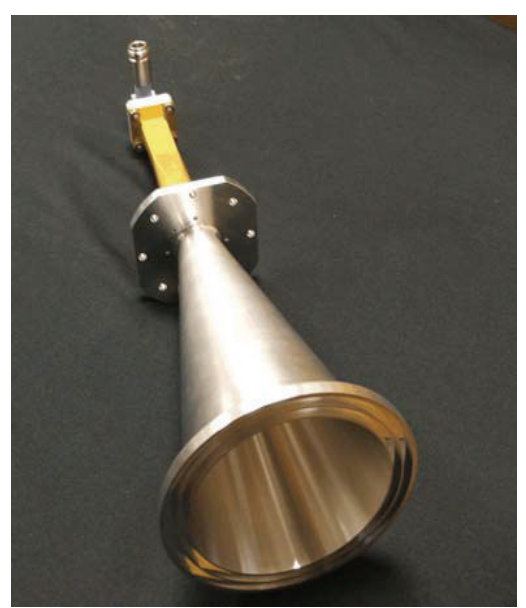

(b)

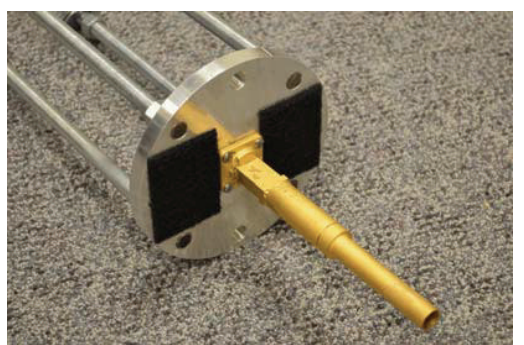

(d)

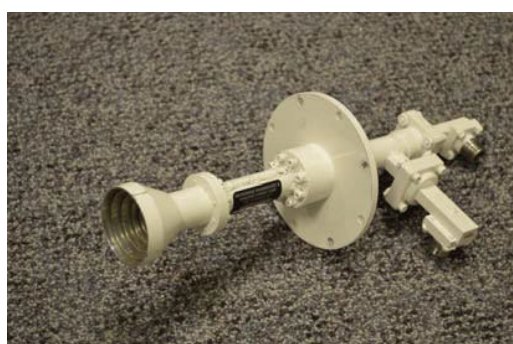

(c)

Figure 3: (a) SN 330. (b) SN 401. (c) SN 1057. (d) SN 331.

Actual probes used in this study are shown in Figure 3. These all operate at Ku band, 12.4-18 GHz.

The plots are normalized so that the peak sensitivity in any figure has the value 1 .

5.1. External Case. Ideal dipole probes provide a direct measurement of components of the electric and magnetic field. Although practical probes only approximate ideal probes at best, ideal probes are effectively assumed in instances when "no probe correction" is applied. Also, practical probes become increasingly ideal as $r_{\mathrm{m}}$ is increased. Figures 46 show sensitivities for a "Huygens probe," which is an ideal probe that consists of a crossed electric and magnetic dipole. This is a maximum-directivity probe of order 1 with a directivity $D=4.77 \mathrm{~dB}[9$, Section 2.3.4]. Figure 4 plots $\alpha_{n}^{\ell}$ as a function of $k r_{\mathrm{m}}$ for several values of $n$. At any given radius, there is very little variation in sensitivity with $n$. Otherwise, we observe the expected decrease in sensitivity as $r_{\mathrm{m}}$ increases. Figures 5 and 6 show $\alpha_{n}^{\ell}$ as a function of $n$ for $k r_{\mathrm{m}}=105$ and 200. When $k r_{\mathrm{m}}=105, c_{\alpha}=1.2$ and at $k r_{\mathrm{m}}=200, c_{\alpha}=1.0$. The trend for conditioning to improve as $r_{\mathrm{m}}$ increases is consistent with an asymptotic analysis of the sensitivity of linearly polarized probes [3, Appendix C].

Figures 7-9 feature the NIST SN $330 \mu= \pm 1$ probe with gain $G=10.5 \mathrm{~dB}$ [3, Appendix B]. Figure 7 plots $\alpha_{n}^{\ell}$ as a function of $k r_{\mathrm{m}}$ for several values of $n$. Compared to the
Huygens probe, there is considerable variation in sensitivity with $n$ for smaller values of $k r_{\mathrm{m}}$. Figures 8 and 9 show $\alpha_{n}^{\ell}$ as a function of $n$ for $k r_{\mathrm{m}}=105$ and 200. When $k r_{\mathrm{m}}=105$, $c_{\alpha}=4.6$, and at $k r_{\mathrm{m}}=200, c_{\alpha}=1.4$.

Figures 10-12 show the NIST SN 1057 dual-port $\mu= \pm 1$ probe with gain $G=15 \mathrm{~dB}$. Figure 10 plots $\alpha_{n}^{\ell}$ as a function of $k r_{\mathrm{m}}$ for several values of $n$. Figures 11 and 12 give $\alpha_{n}^{\ell}$ as a function of $n$ for $k r_{\mathrm{m}}=105$ and 200. When $k r_{\mathrm{m}}=105, c_{\alpha}=$ 19 , and at $k r_{\mathrm{m}}=200, c_{\alpha}=3.1$.

Figures 13-15 feature the NIST SN $401 \mu= \pm 1$ probe with gain $G=22.7 \mathrm{~dB}$. This probe resembles SN 330, except that a flare has been added to increase the directivity. Figure 13 plots $\alpha_{n}^{\ell}$ as a function of $k r_{\mathrm{m}}$ for several values of $n$. Variation in sensitivity with $n$ for smaller values of $k r_{\mathrm{m}}$ is problematic. Figures 14 and 15 show $\alpha_{n}^{\ell}$ as a function of $n$ for $k r_{\mathrm{m}}=120$ and 500. When $k r_{\mathrm{m}}=120, c_{\alpha}=200$ and at $k r_{\mathrm{m}}=500, c_{\alpha}=2.8$. In order to obtain similar conditioning, this probe must be used at considerably larger values of $r_{\mathrm{m}}$ than those for the lower directivity probes discussed here.

5.2. Internal Case. In the internal case, conditioning is significantly affected by the fact that radial functions (spherical Bessel functions) have zeros. Figure 16 plots $\alpha_{n}^{\ell}$ as a function of $k r_{\mathrm{m}}$ for several values of $n$, when the probe is an ideal electric dipole $(D=1.76 \mathrm{~dB})$. The variation in sensitivity with $n$ is extreme and it seems unlikely that any choice of 


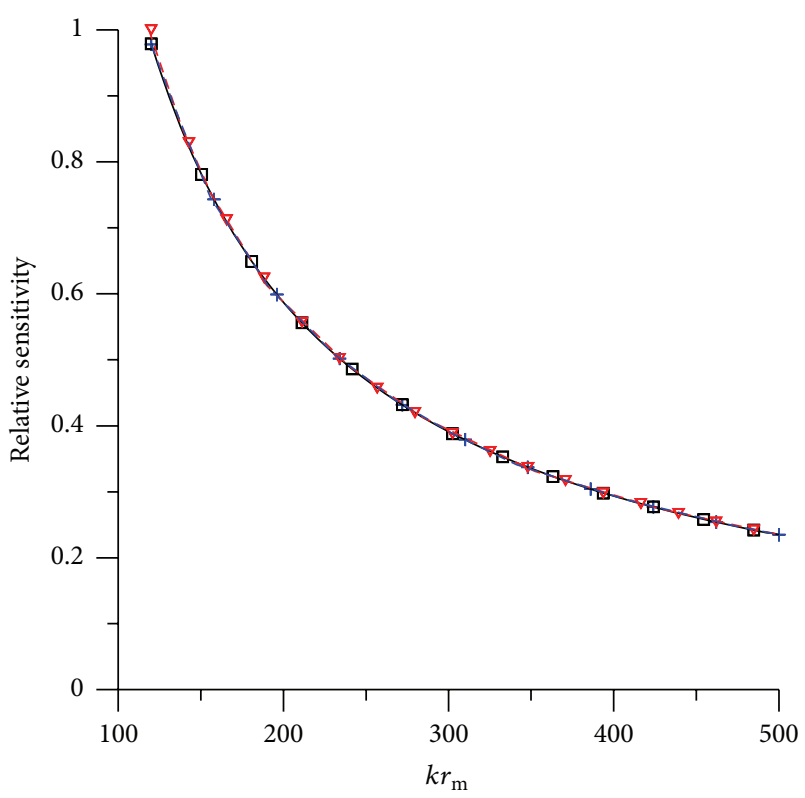

Huygens probe

$-+-n=10$, magnetic, electric

$\square n=50$, magnetic, electric

$-\nabla-n=90$, magnetic, electric

Figure 4: Huygens probe, external case.

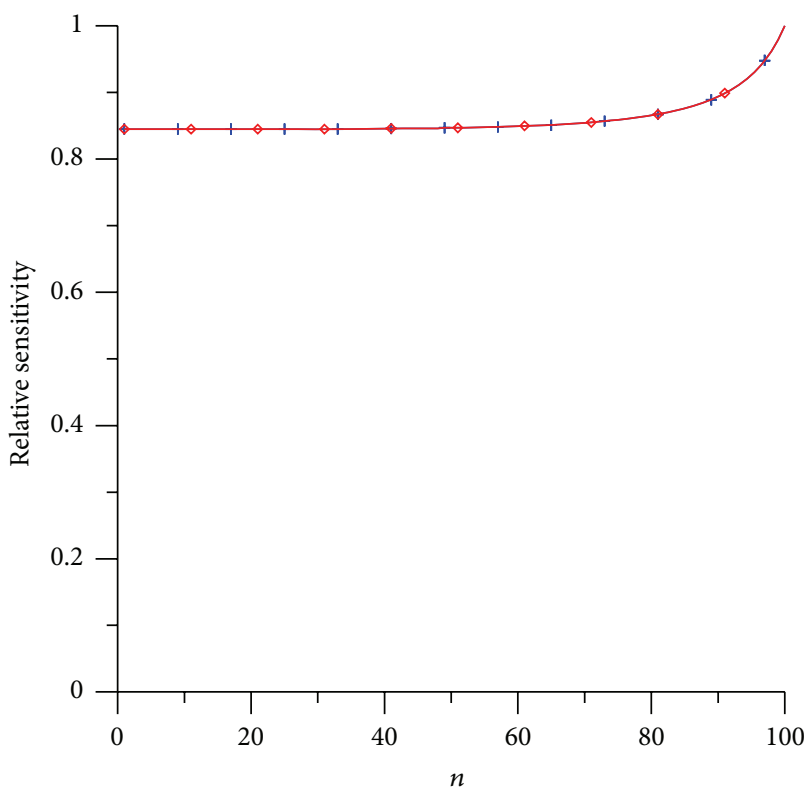

Huygens probe + Magnetic

$\diamond$ Electric

FIGURE 5: Huygens probe, external case, $k r_{\mathrm{m}}=105$.

one or several values of $r_{\mathrm{m}}$ would allow reliable determination of all of the modes. Figure 17 plots $\alpha_{n}^{\ell}$ as a function of $n$ for $k r_{\mathrm{m}}=104.6$. At this radius, $c_{\alpha}=830$.

Fortunately, the Huygens probe $(D=4.77 \mathrm{~dB})$ is much better behaved, as illustrated in Figures 18 and 19. Figure 18

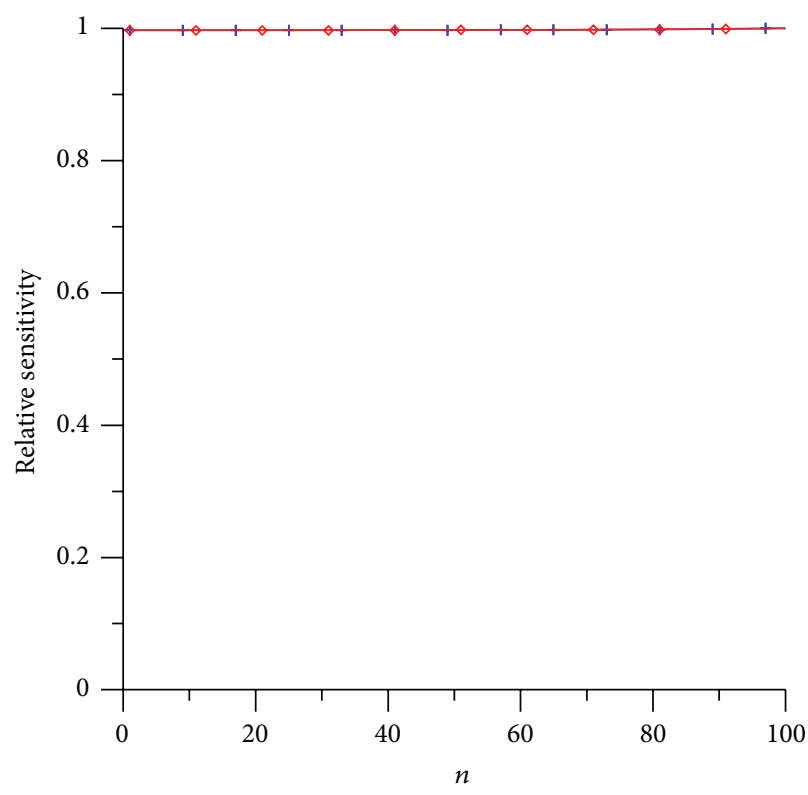

$$
\begin{aligned}
& \text { Huygens probe } \\
& + \text { Magnetic } \\
& \triangleleft \text { Electric }
\end{aligned}
$$

FIGURE 6: Huygens probe, external case, $k r_{\mathrm{m}}=200$.

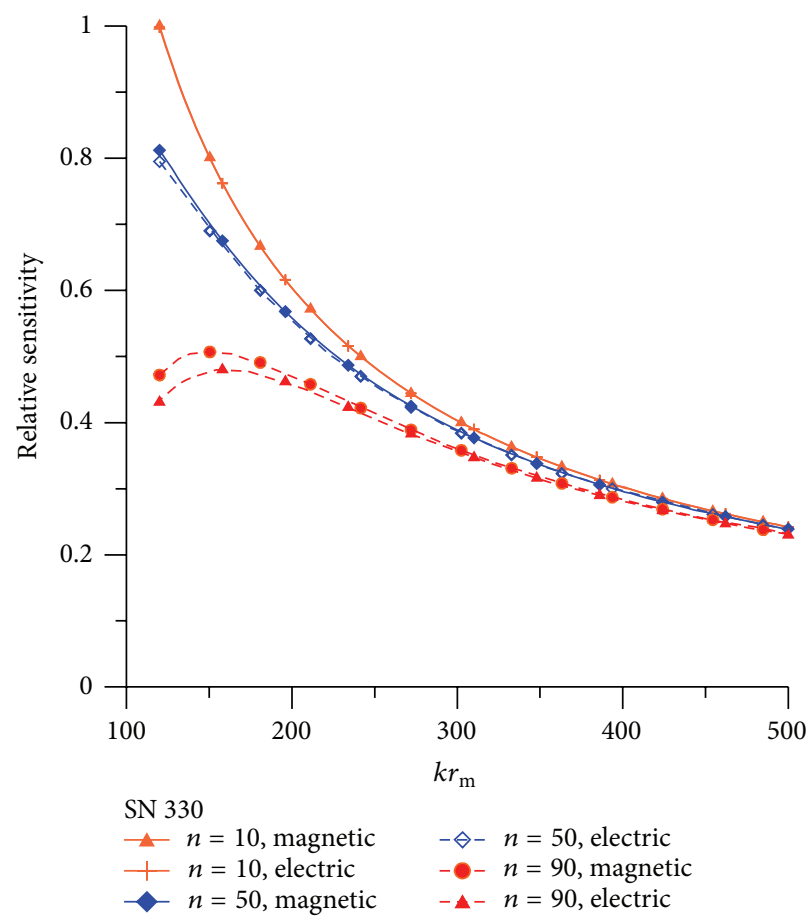

Figure 7: SN 330, external case.

plots $\alpha_{n}^{\ell}$ as a function of $k r_{\mathrm{m}}$ for several values of $n$ and indicates a tendency for conditioning to improve as $r_{\mathrm{m}}$ increases. Figure 19 shows $\alpha_{n}^{\ell}$ as a function of $n$ for $k r_{\mathrm{m}}=106$. At this radius, $c_{\alpha}=2.8$.

Figures 20 and 21 feature the NIST SN 330 probe $(G=$ $10.5 \mathrm{~dB}$ ). Figure 20 plots $\alpha_{n}^{\ell}$ as a function of $k r_{\mathrm{m}}$ for several 


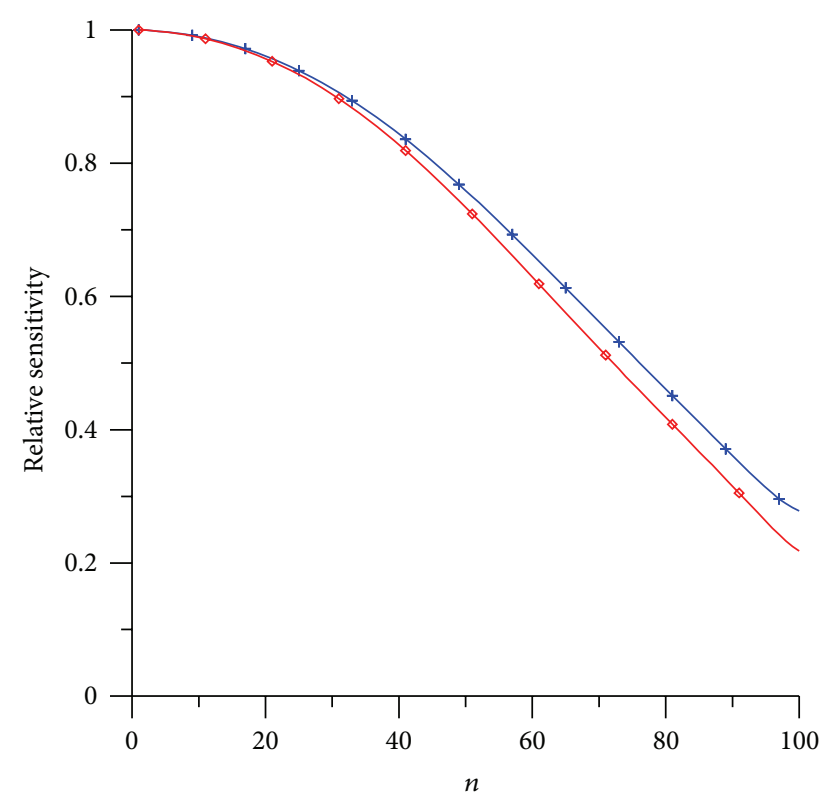

SN 330

+ Magnetic

$\diamond$ Electric

FIGURE 8: SN 330, external case, $k r_{\mathrm{m}}=105$.

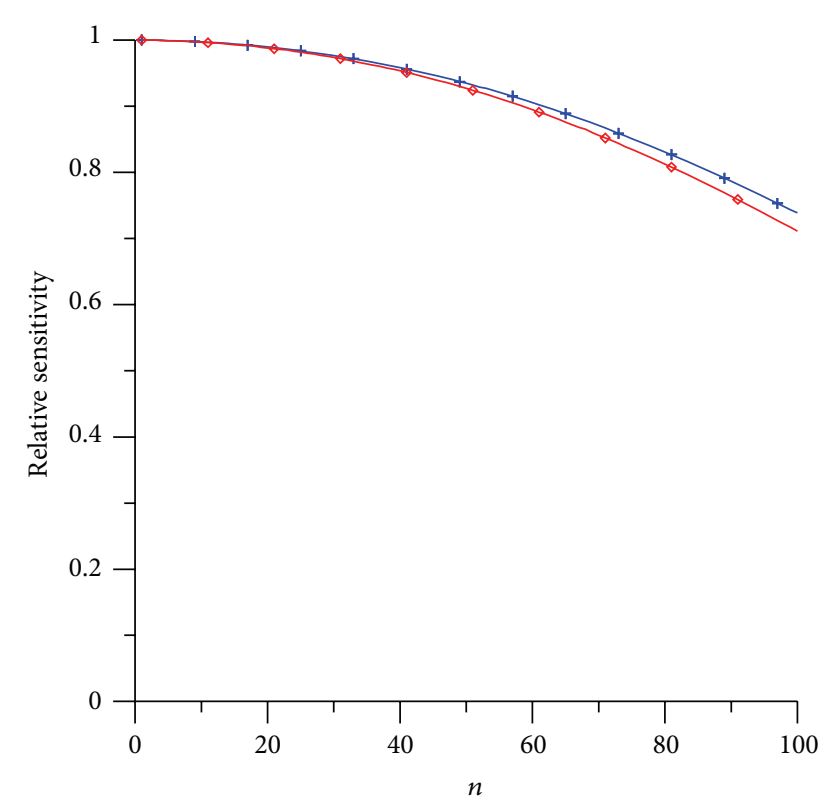

SN 330
+ Magnetic
$\triangleleft$ Electric

Figure 9: SN 330, external case, $k r_{\mathrm{m}}=200$.

values of $n$. Again, conditioning appears to improve as measurement radius is increased. Figure 21 shows $\alpha_{n}^{\ell}$ as a function of $n$ for $k r_{\mathrm{m}}=110$, for which $c_{\alpha}=4.2$.

Figures 22 and 23 show the NIST SN 1057 dual-port probe ( $G=15 \mathrm{~dB})$. Figure 22 plots $\alpha_{n}^{\ell}$ as a function of $k r_{\mathrm{m}}$ for several values of $n$. Again, conditioning appears to improve

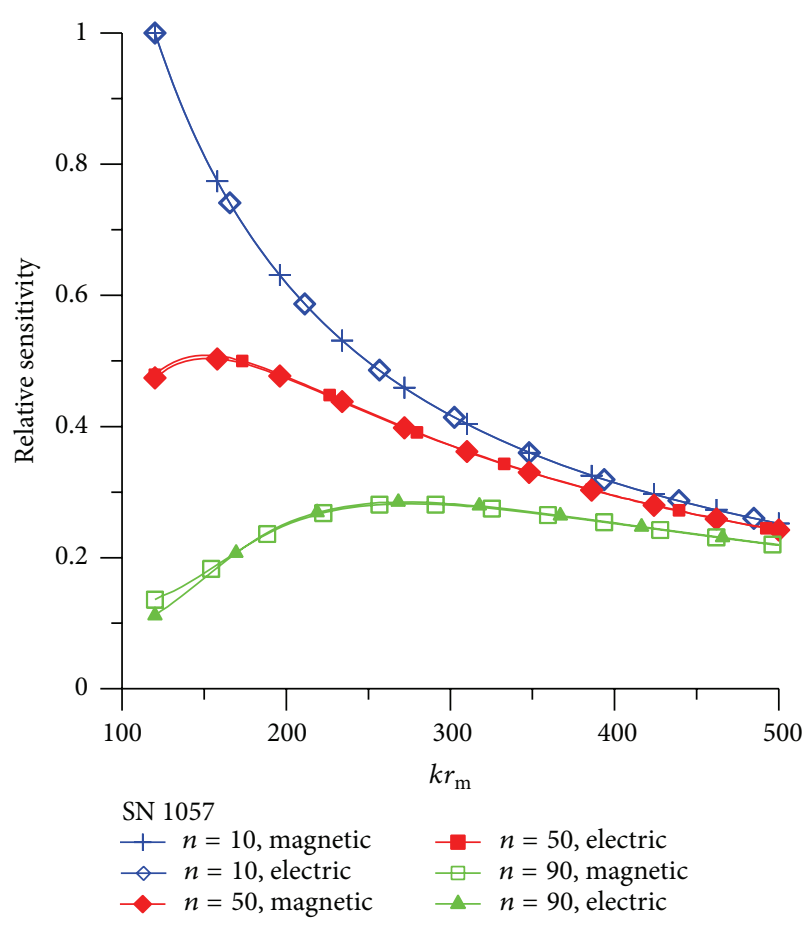

FIGURE 10: SN 1057, external case.

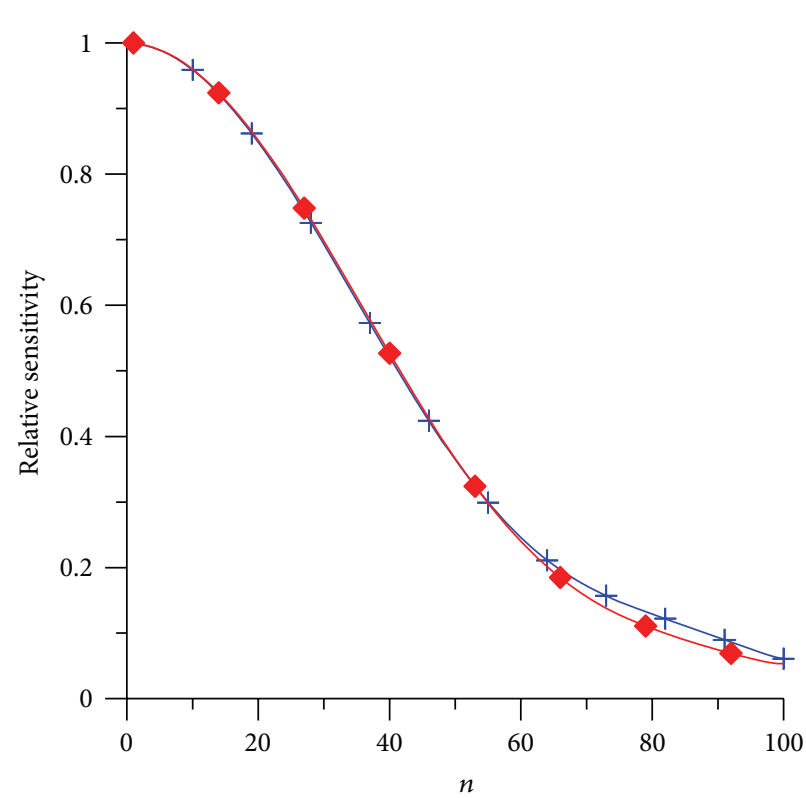

SN 1057

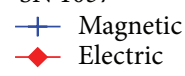

FIGURE 11: SN 1057, external case, $k r_{\mathrm{m}}=105$.

as measurement radius is increased. Figure 23 shows $\alpha_{n}^{\ell}$ as a function of $n$ for $k r_{\mathrm{m}}=110$, for which $c_{\alpha}=20$.

Figures 24 and 25 feature the NIST SN 401 probe ( $G=$ $22.7 \mathrm{~dB}$ ). Figure 24 plots $\alpha_{n}^{\ell}$ as a function of $k r_{\mathrm{m}}$ for several values of $n$ and indicates a significant range of sensitivity with $n$. Figure 25 shows $\alpha_{n}^{\ell}$ as a function of $n$ for $k r_{\mathrm{m}}=110$, for 


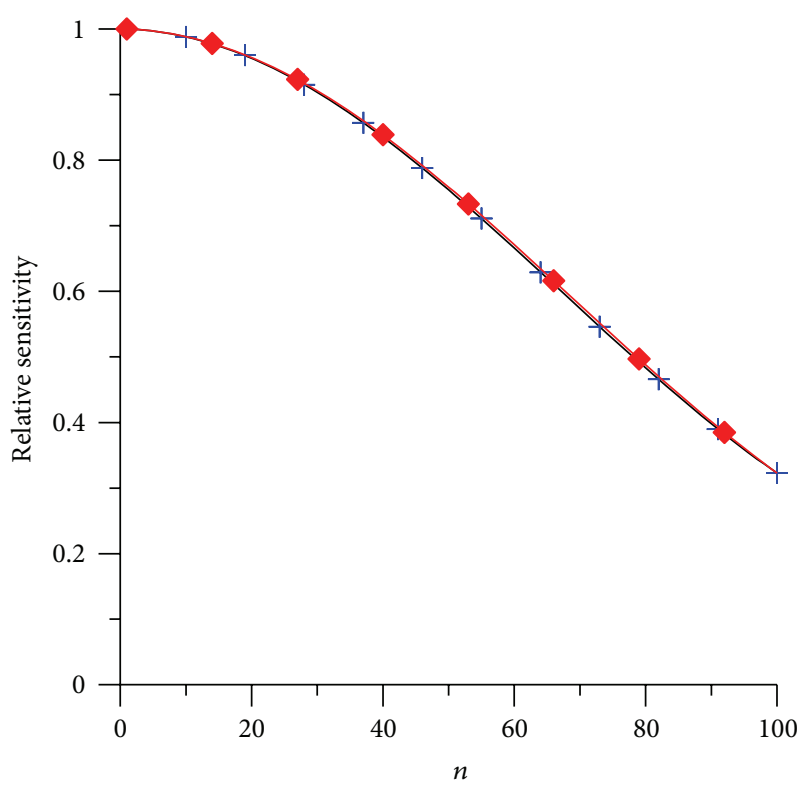

SN 1057

+ Magnetic

FIgURE 12: SN 1057, external case, $k r_{\mathrm{m}}=200$.

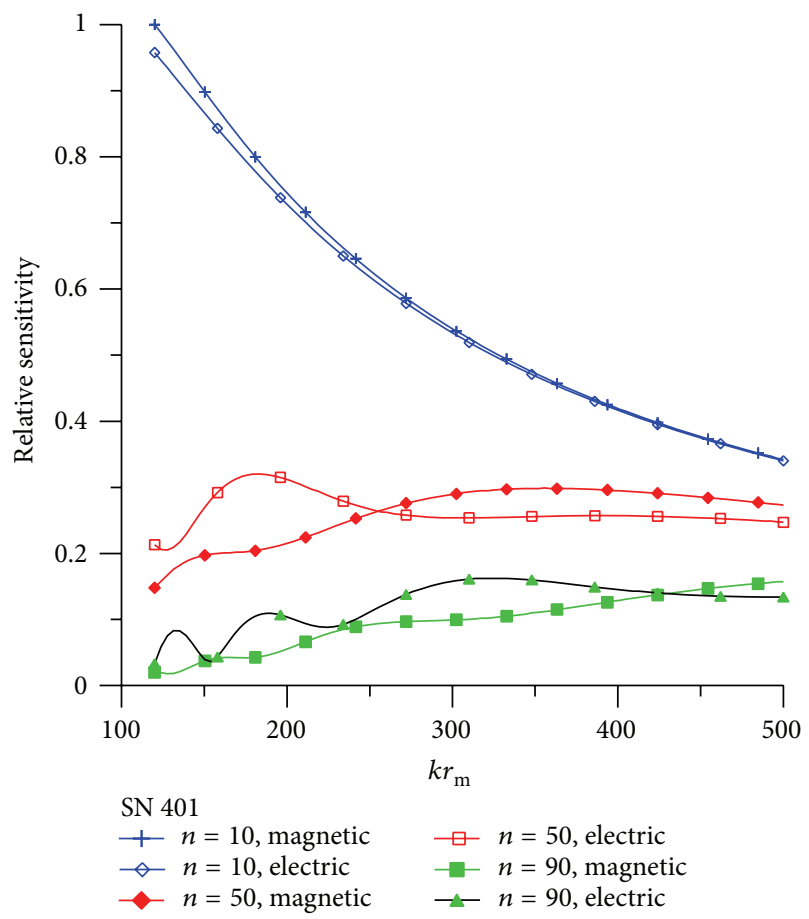

Figure 13: SN 401, external case.

which $c_{\alpha}=2200$. This is certainly unacceptable for many applications.

Figure 26 compares NIST SN $330(G=10.5 \mathrm{~dB})$, SN 331 $(G=7.9 \mathrm{~dB})$, and SN $401(G=22.7 \mathrm{~dB})$ at $k r_{\mathrm{m}}=110$. SN 331 is a version of SN 330 with a smaller aperture. With all things equal, SN 401 has better sensitivity for multipole indexes less

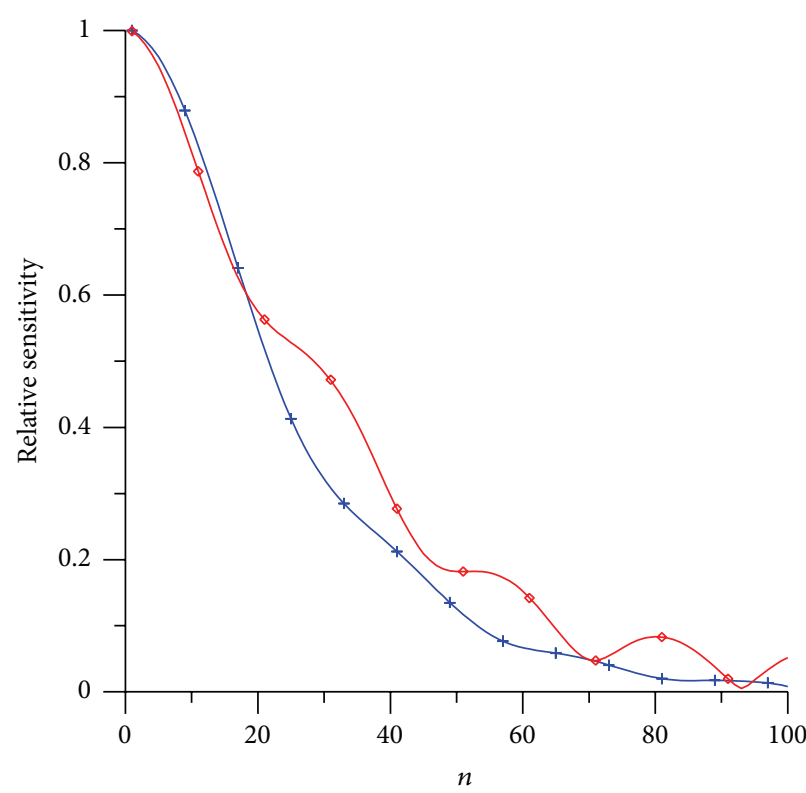

SN 401

+ Magnetic

$\diamond$ Electric

FIGURE 14: SN 401, external case, $k r_{\mathrm{m}}=120$.

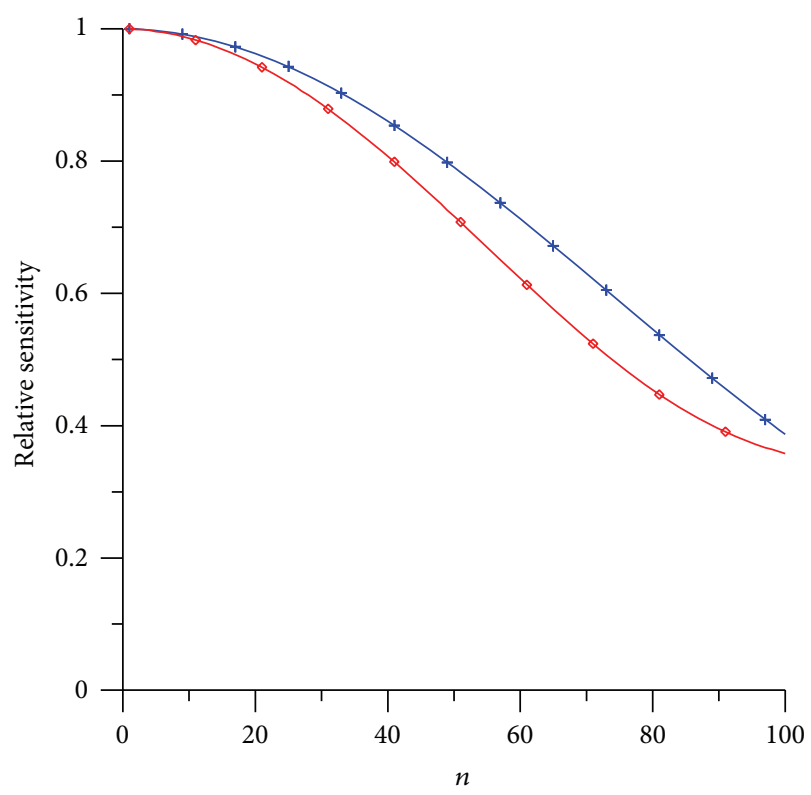

SN 401

+ Magnetic

$\diamond$ Electric

FIGURE 15: SN 401, external case, $k r_{\mathrm{m}}=500$.

than $n \approx 20$ but is significantly less sensitive for larger values of $n$. Because of its higher gain, however, the SN 401 must be used at lower transmitted power levels. Improving the signalto-noise ratio by increasing the transmitted power will tend to compensate for the reduced sensitivity of the lower-gain probes when $n<20$. 


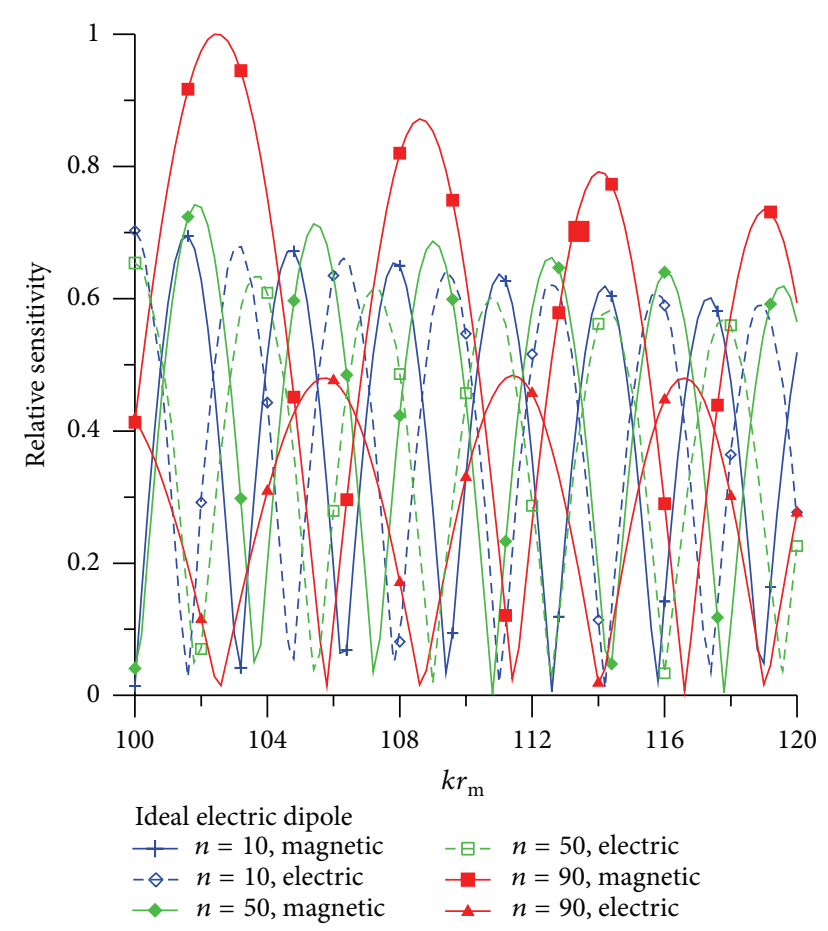

FIgURE 16: Ideal electric dipole, internal case.

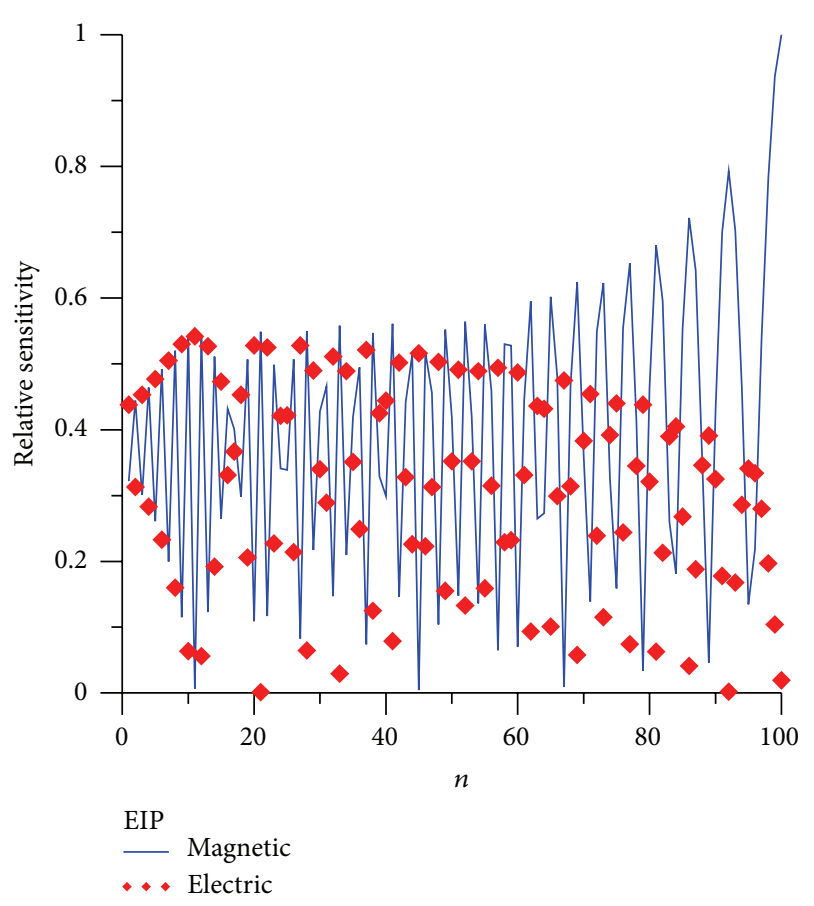

FIGURE 17: Ideal electric dipole, $k r_{\mathrm{m}}=104.6$.

\section{Conclusions}

For any given probe, sensitivities generally become more uniform as the measurement radius is increased. More directive probes must be used at larger measurement radii

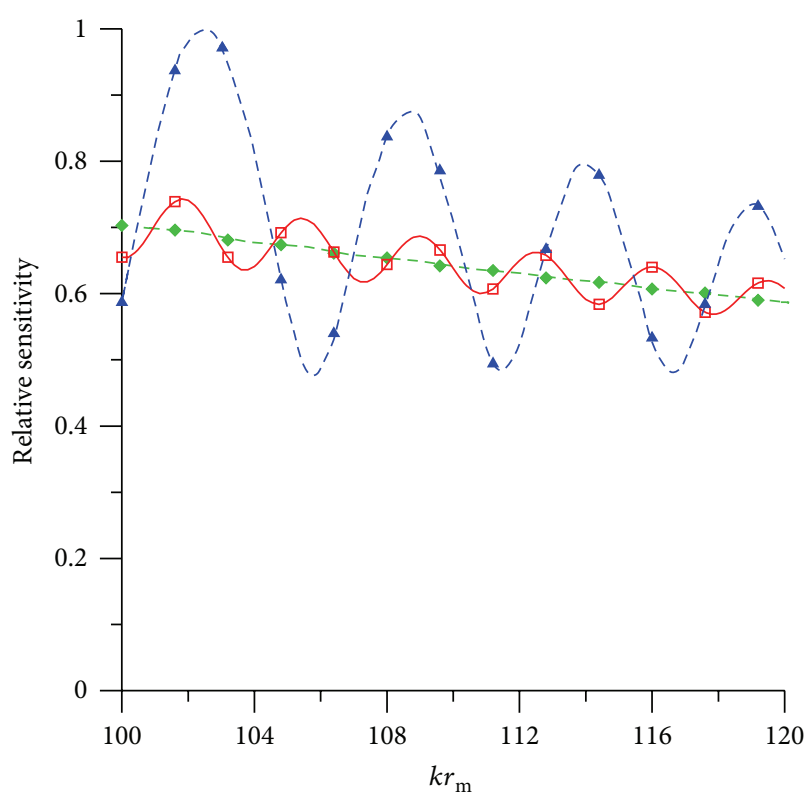

Huygens probe

$-\diamond-n=10$, magnetic, electric

$\square n=50$, magnetic, electric

$-\Delta-n=90$, magnetic, electric

FIGURE 18: Huygens probe, internal case.

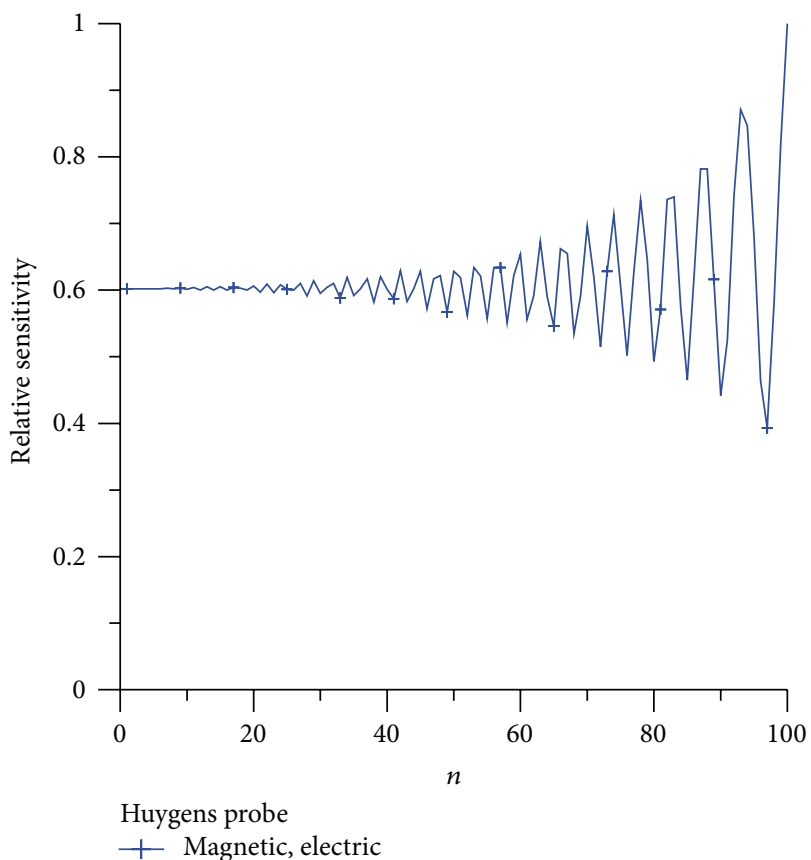

Figure 19: Huygens probe, internal case, $k r_{\mathrm{m}}=106$.

than less directive probes if the same condition number is desired.

The topic of probe sensitivity in spherical scanning seems not to have received much attention to date; yet, sensitivity can have a very significant impact on measurement accuracy. Our results are based on a limited number of examples; however, sensitivity information is readily available to anyone 


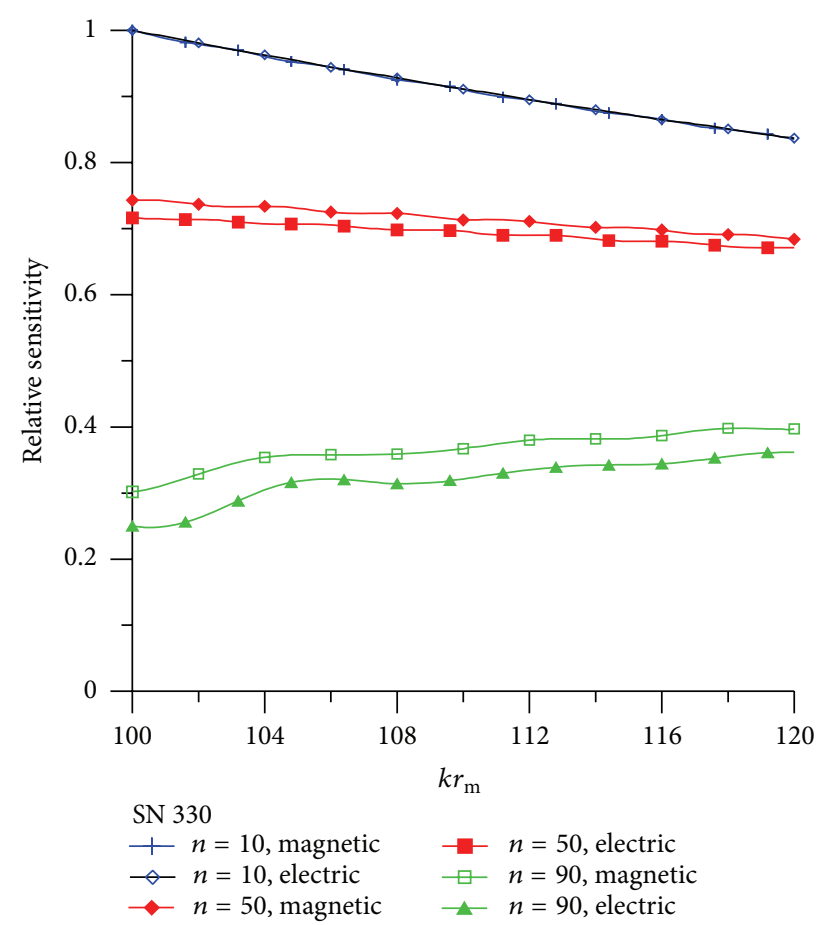

Figure 20: SN 330, internal case.

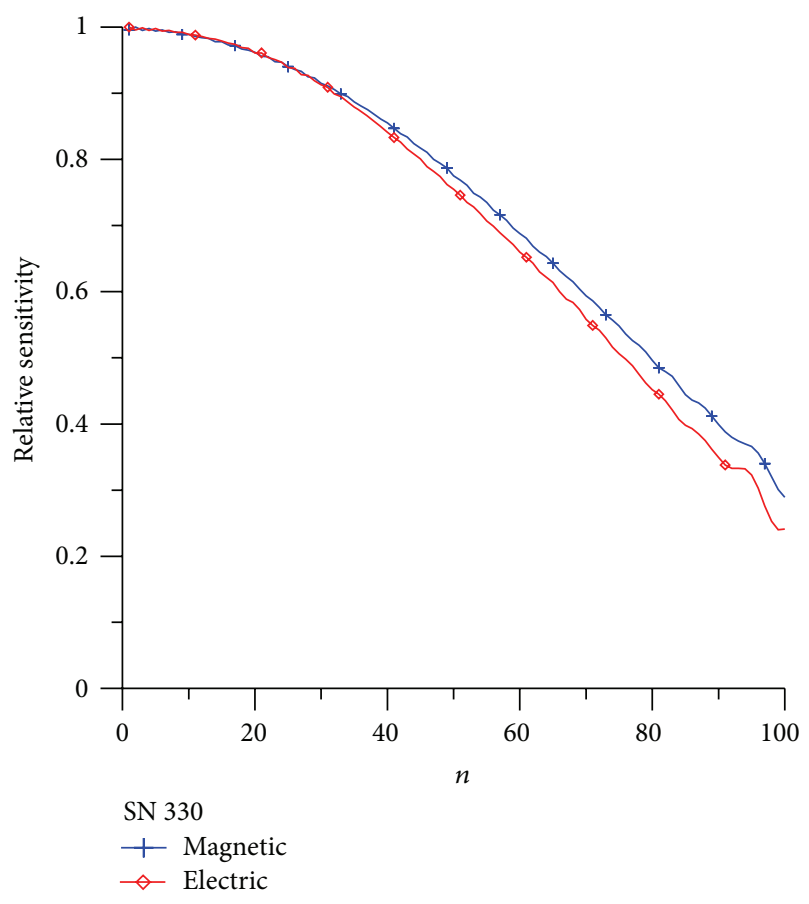

FIGURE 21: SN 330, internal case, $k r_{\mathrm{m}}=110$.

who processes spherical-scanning data. We strongly encourage metrologists to explore probe-sensitivity issues before making near-field antenna measurements. Optimization of sensitivity should be a consideration in the design of new probes.

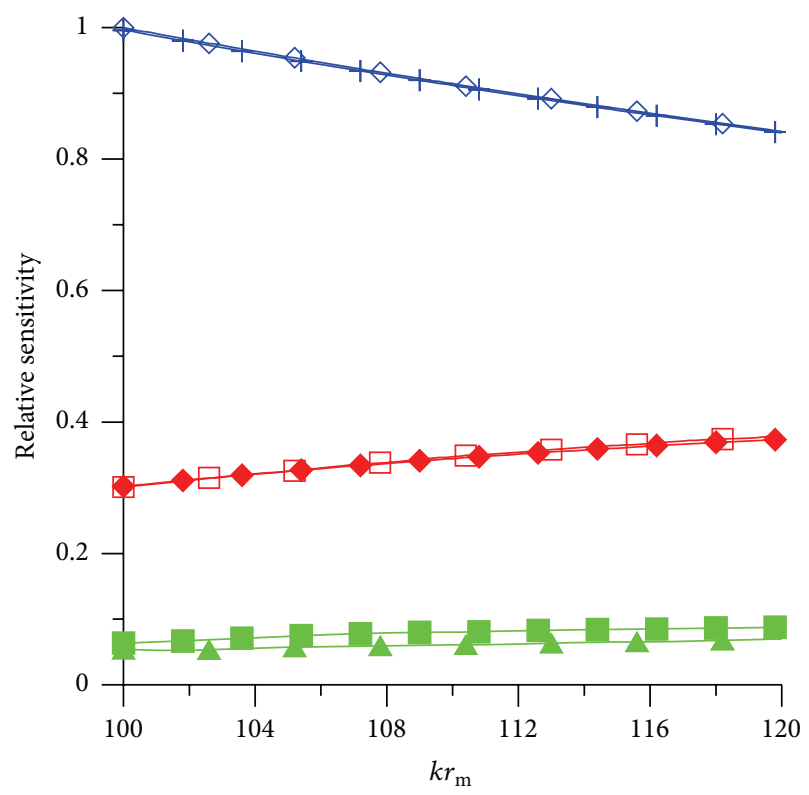

$$
\begin{aligned}
& \text { SN } 1057 \\
& \because n=10 \text {, magnetic } \square n=50 \text {, electric } \\
& \diamond n=10 \text {, electric } \rightarrow n=90 \text {, magnetic } \\
& \multimap n=50 \text {, magnetic } \multimap n=90 \text {, electric }
\end{aligned}
$$

FIGURE 22: SN 1057, internal case.

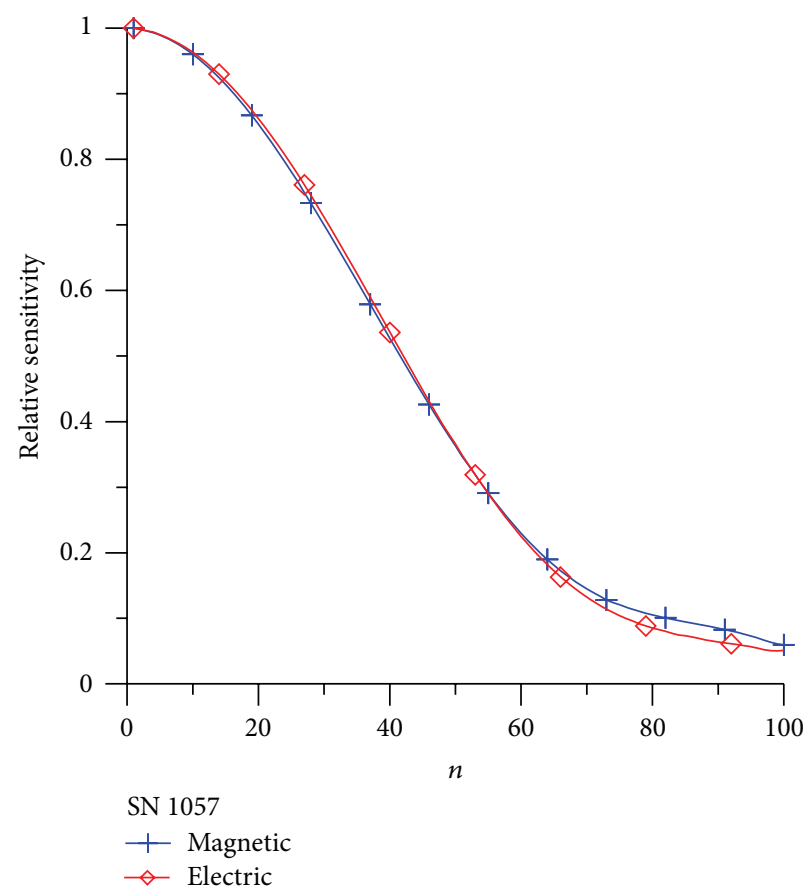

FIGURE 23: SN 1057, internal case, $k r_{\mathrm{m}}=110$.

\section{Appendix}

\section{Partial Analysis of Uncertainty}

From (8)

$$
T_{n}=\alpha_{n} t_{n},
$$

where we are using a compact notation. 


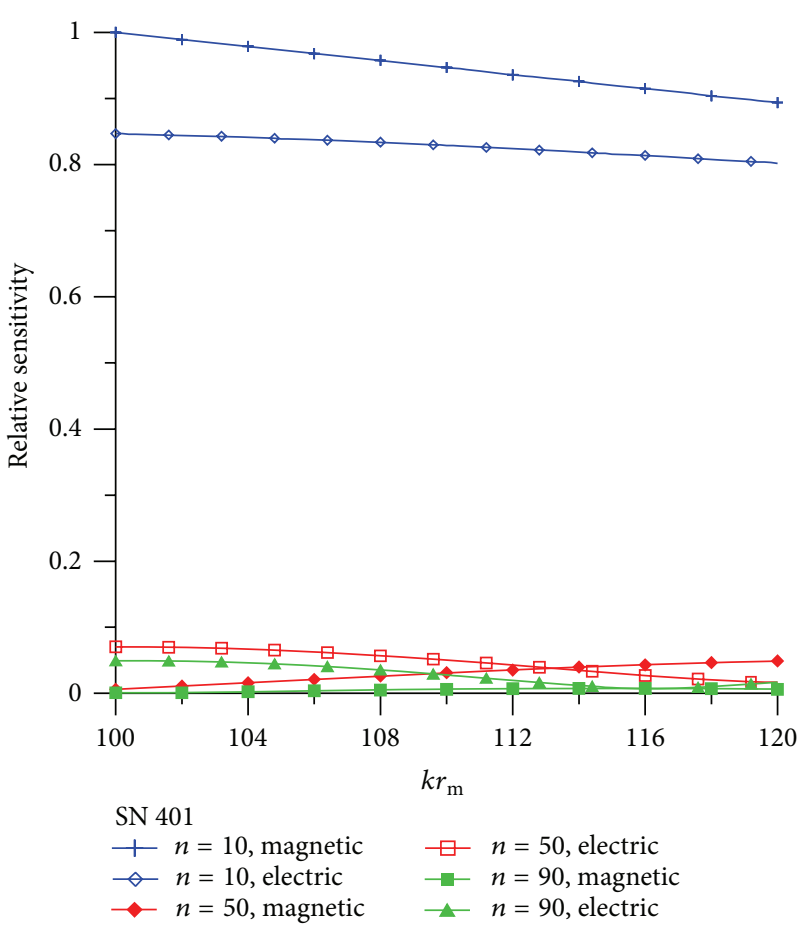

FIGURE 24: SN 401, internal case.

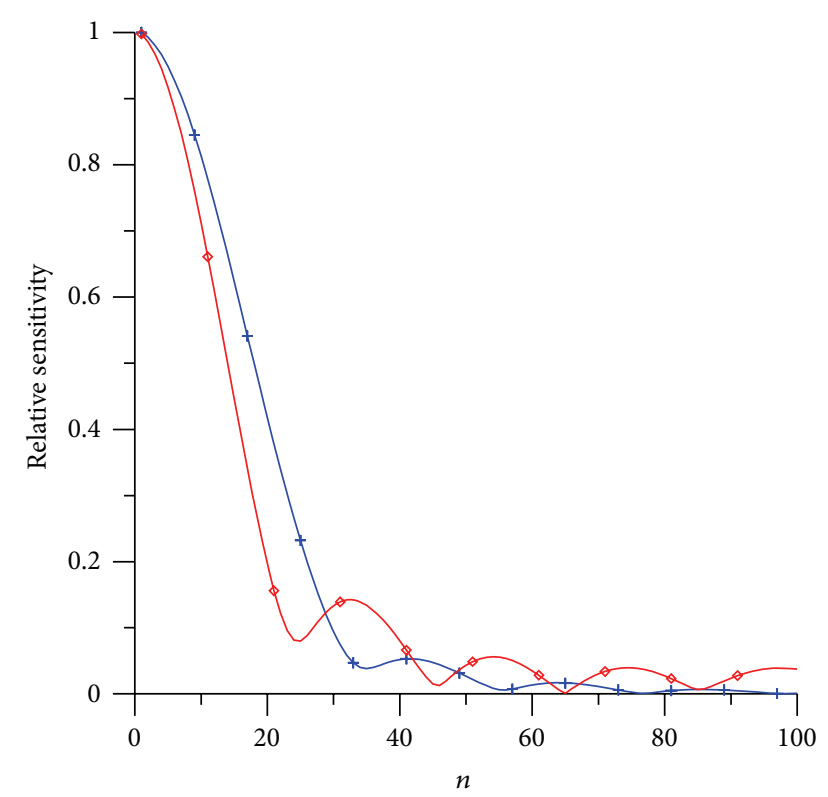

SN 401

+ Magnetic
$\neg$ Electric

FIGURE 25: SN 401, internal case, $k r_{\mathrm{m}}=110$.

When $\Delta \alpha_{n}=0$,

$$
\begin{aligned}
t_{n} & =\frac{T_{n}}{\alpha_{n}} \\
\Delta t_{n} & =\frac{\Delta T_{n}}{\alpha_{n}},
\end{aligned}
$$

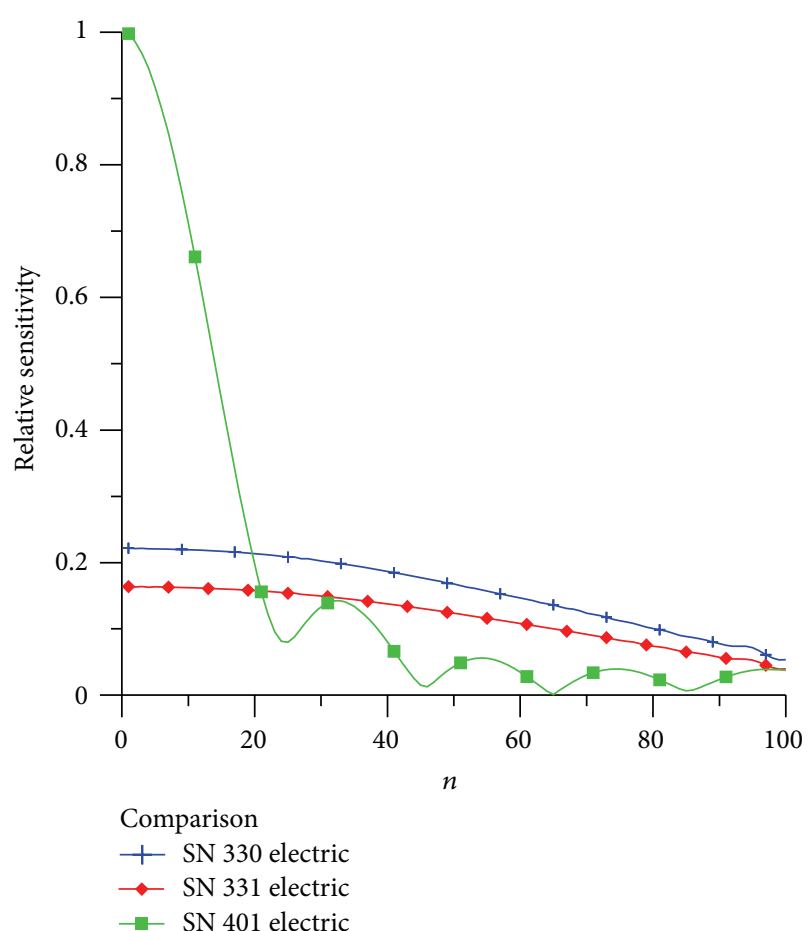

FIGURE 26: Comparison, internal case, $k r_{\mathrm{m}}=110$.

$$
\begin{gathered}
\|\mathbf{t}\|^{2}=\sum_{n}\left|\frac{T_{n}}{\alpha_{n}}\right|^{2} \geq \frac{1}{\left|\alpha_{\max }\right|^{2}}\|\mathbf{T}\|^{2} \\
\|\Delta \mathbf{t}\|^{2}=\sum_{n}\left|\frac{\Delta T_{n}}{\alpha_{n}}\right|^{2} \leq \frac{1}{\left|\alpha_{\min }\right|^{2}}\|\Delta \mathbf{T}\|^{2} .
\end{gathered}
$$

Therefore,

$$
\frac{\|\Delta \mathbf{t}\|}{\|\mathbf{t}\|} \leq\left|\frac{\alpha_{\max }}{\alpha_{\min }}\right| \frac{\|\Delta \mathbf{T}\|}{\|\mathbf{T}\|}=c_{\alpha} \frac{\|\Delta \mathbf{T}\|}{\|\mathbf{T}\|} .
$$

When $\Delta T_{n}=0$,

$$
\begin{gathered}
\Delta t_{n}=\alpha_{n} \Delta\left(\frac{1}{\alpha_{n}}\right) t_{n}, \\
\|\Delta \mathbf{t}\|^{2}=\sum_{n}\left|\alpha_{n} \Delta\left(\frac{1}{\alpha_{n}}\right) t_{n}\right|^{2} \leq \beta^{2}\|\mathbf{t}\|^{2} .
\end{gathered}
$$

Therefore,

$$
\begin{gathered}
\frac{\|\Delta \mathbf{t}\|}{\|\mathbf{t}\|} \leq \beta \\
\beta_{\max }=\max _{n}\left|\alpha_{n} \Delta\left(\frac{1}{\alpha_{n}}\right)\right| \approx \max _{n}\left|\frac{\Delta \alpha_{n}}{\alpha_{n}}\right| .
\end{gathered}
$$




\section{Disclosure}

This research is US government work not protected by US copyright.

\section{Conflict of Interests}

The authors declare that there is no conflict of interests regarding the publication of this paper.

\section{References}

[1] A. C. Newell, "Error analysis techniques for planar near-field measurements," IEEE Transactions on Antennas and Propagation, vol. 36, no. 6, pp. 754-768, 1988.

[2] R. C. Wittmann and M. H. Francis, "Probe sensitivity in nearfield, spherical-scanning, antenna measurements," in Proceedings of the AMTA, pp. 15-20, Bellevue, Wash, USA, October 2012.

[3] M. H. Francis and R. C. Wittmann, "Near-field scanning measurements: theory and practice," in Modern Antenna Handbook, C. A. Balanis, Ed., chapter 19, John Wiley \& Sons, New York, NY, USA, 2008.

[4] J. D. Jackson, Classical Electrodynamics, John Wiley \& Sons, New York, NY, USA, 2nd edition, 1975.

[5] R. C. Wittmann and M. H. Francis, "Spherical scanning measurements: propagating errors through the near-to far-field transformation," in Proceedings of the AMTA, pp. 74-79, Atlanta, Ga, USA, October 2004.

[6] R. C. Wittmann, "Spherical near-field scanning: determining the incident field near a rotatable probe," in Proceedings of the IEEE 1990 Antennas and Propagation Society International Symposium, pp. 224-227, May 1990.

[7] D. N. Black Jr. and E. B. Joy, "Test zone field compensation," IEEE Transactions on Antennas and Propagation, vol. 43, no. 4, pp. 362-368, 1995.

[8] D. A. Leatherwood and E. B. Joy, "Plane wave, pattern subtraction, range compensation," IEEE Transactions on Antennas and Propagation, vol. 49, no. 12, pp. 1843-1851, 2001.

[9] J. E. Hansen, Ed., Spherical Near-Field Antenna Measurements, Peregrinus, London, UK, 1988. 

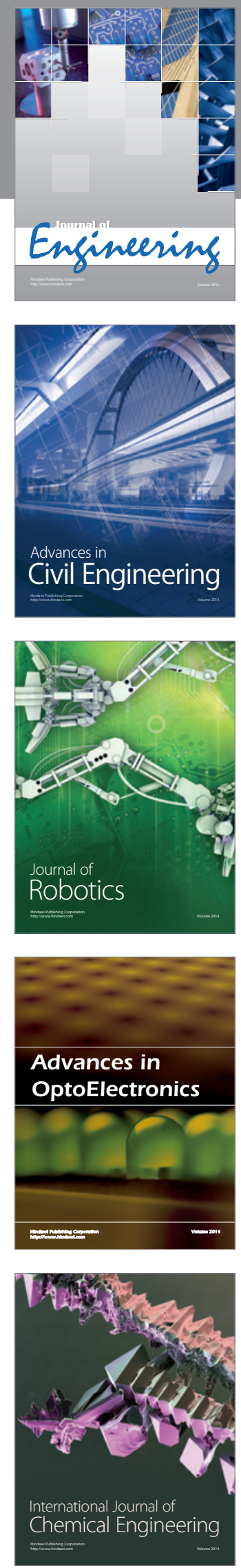

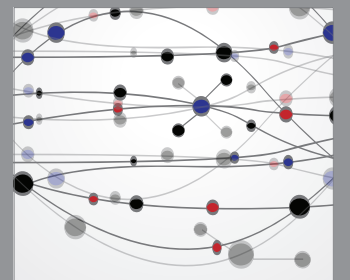

The Scientific World Journal
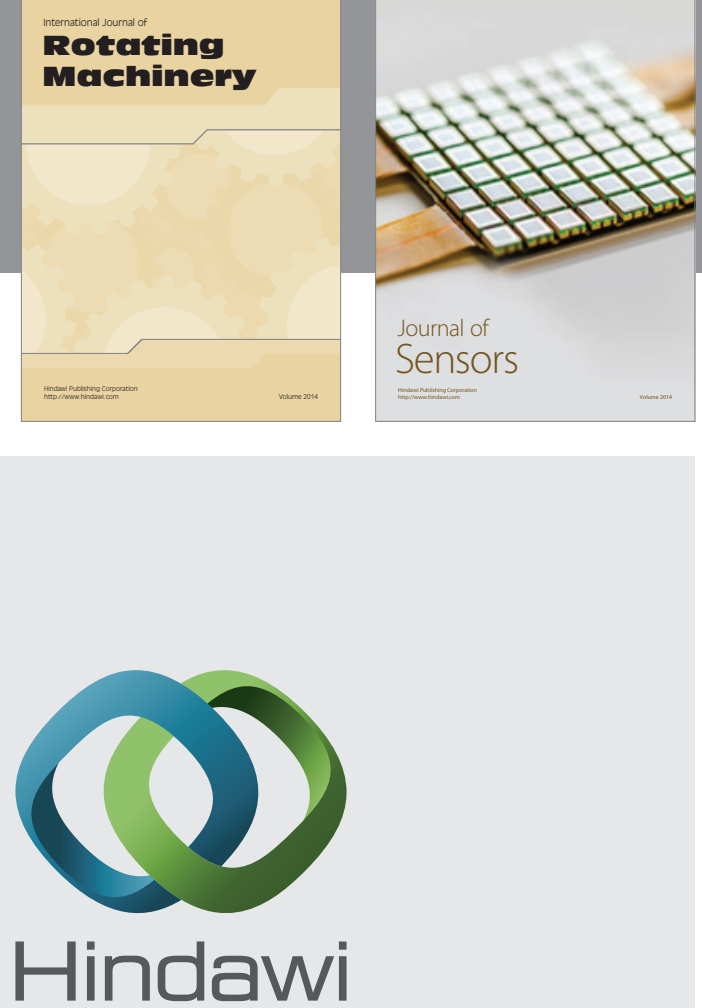

Submit your manuscripts at http://www.hindawi.com
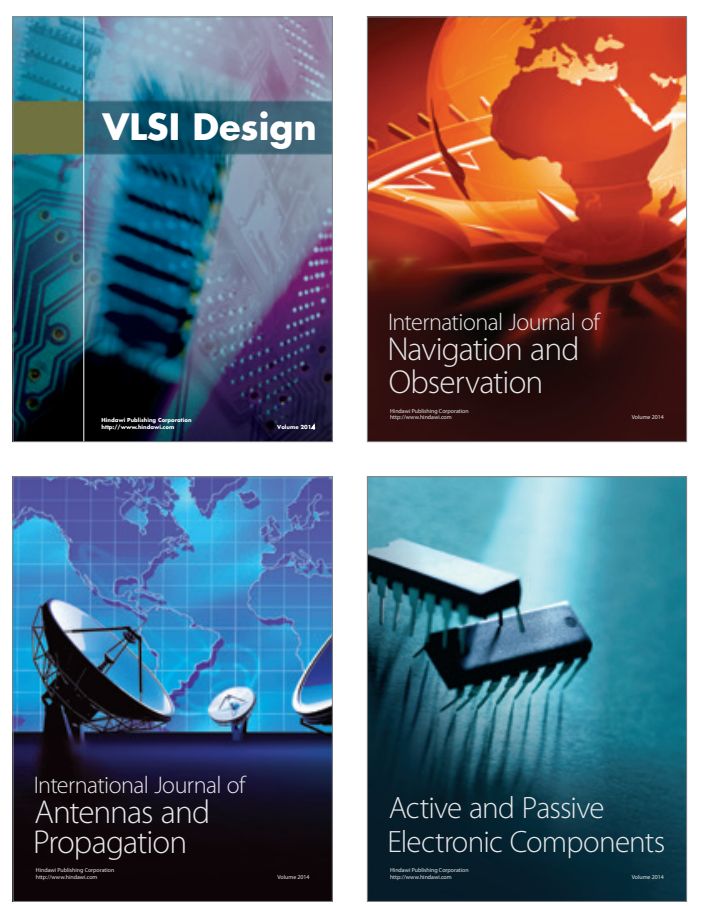
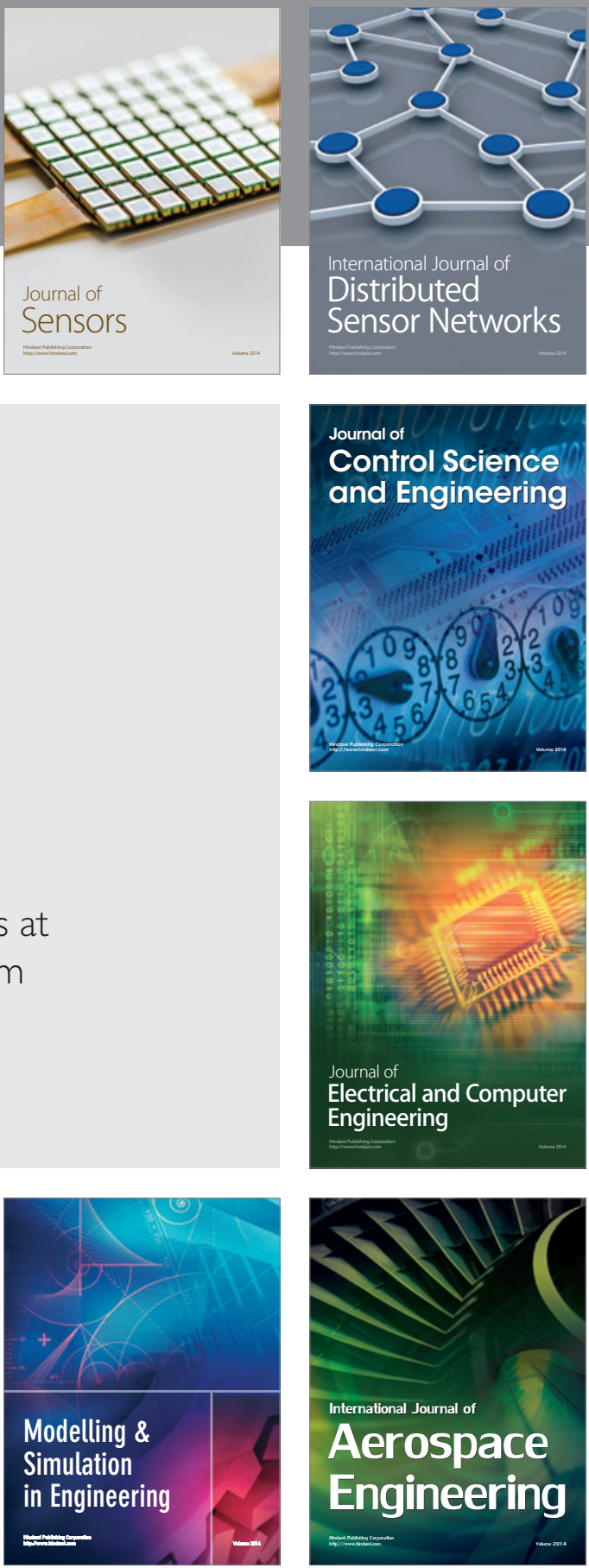

Journal of

Control Science

and Engineering
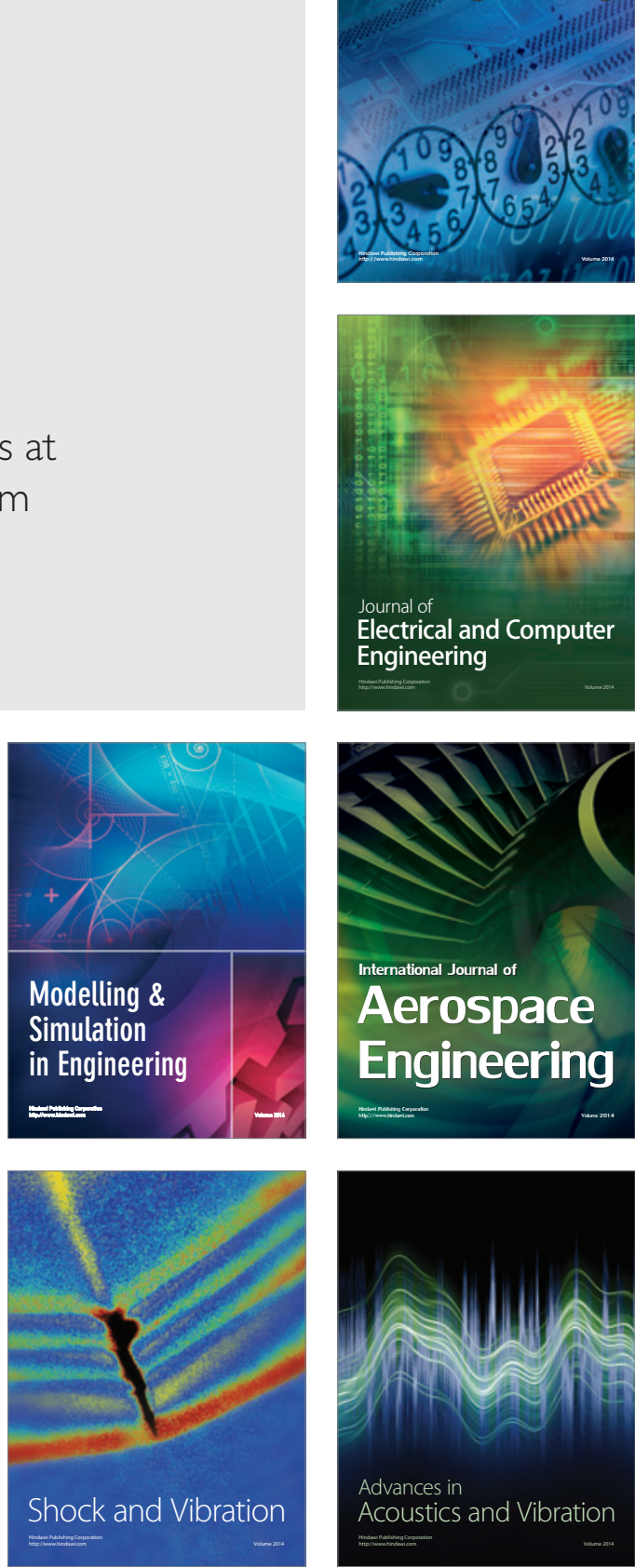